\title{
COMPARISON OF DIFFERENT MACHINE LEARNING APPROACHES TO MODEL STROKE SUBTYPE CLASSIFICATION AND RISK PREDICTION
}

\author{
Luis García-Terriza \\ José L. Risco-Martín \\ José L. Ayala \\ Department of Computer Architecture \\ and Automation \\ Universidad Complutense de Madrid \\ Madrid, Spain \\ \{luigar05,jlrisco,jayala\}@ucm.es
}

\author{
Gemma Reig Roselló \\ Juan M. Camarasaltas \\ Hospital Universitario de La Princesa \\ Madrid, Spain \\ gemmareig@hotmail.com \\ juanmiguel.camarasaltas@salud.madrid.org
}

\begin{abstract}
The application of computer-aided techniques regarding stroke diagnosis is especially important in nonurban areas, because of the lack of adequate resources. The purpose of this research consists of testing the hypothesis that state-of-the-art machine learning-based modeling techniques, when integrated with noninvasive monitoring technologies, can help on the diagnosis of the type of stroke only few minutes after the crisis. This method has been also tested to predict future risks, like the eventual death of the patient.

The collected dataset comprised the medical records of 119 patients with 7 predictors and two target variables: diagnosis of stroke type and prediction of death. 7 different algorithms have been employed, evaluated over 6 different metrics. 10-fold cross validation resampling method was utilized. Random Forest models produced the best performance in stroke diagnosis and death prediction compared to the other algorithms, with average values of $0.93 \pm 0.03$ and $0.97 \pm 0.01$, respectively.
\end{abstract}

Keywords: Stroke, Machine Learning, Supervised Classification

\section{INTRODUCTION AND RELATED WORK}

Techniques based on Artificial Intelligence (AI) are recently invading health-care, even injecting the idea of whether AI doctors will eventually replace human physicians in the future. As in (Jiang et al. 2017), we truly believe that human physicians will not be replaced by machines in a distant future. However, AI can assist to make better clinical decisions by quickly performing data analysis before a complex test. In this paper, we apply this idea to the stroke case of study.

Stroke is one of the main causes of death in the world and also a great cause of serious and long-term disabilities (Haidong Wang 2016). There are two types of stroke: hemorrhagic and ischemic. The first ones are provoked by vessel rupture, while the second ones are caused by blood clots occluding brain arteries (Alexopoulos, Dounias, and Vemmos 1999). According to the World Health Organization, stroke is the cause of 6.2 millions of deaths each year, being the second leading cause of death and the third leading cause of disability (World Health Organization 2018). 
Stroke is diagnosed through several techniques: neurological examination, Computed Tomography (CT), Magnetic Resonance Imaging (MRI), Doppler ultrasound, and arteriography. The diagnosis of stroke itself is clinical, with assistance from the imaging exams. Imaging techniques also assist in determining the subtypes and cause of stroke. In the case of stroke diagnosis, time is critical. Earlier treatment gives better outcomes (Hill 2005). Being able to identify ischemic vs hemorrhagic stroke is mandatory in order to apply the suitable treatment. Not only to diagnose, but also to predict future medical complications. In this regard, computer-aided design methods can help to diagnose or predict future risks. Kanchana and Menaka in (Kanchana and Menaka 2017), for instance, use computed aided design for the characterization of ischemic stroke lesion segmenting the tomography image.

However, imaging techniques are not accessible to everyone. The importance of time-saving and accurate methods for stroke diagnosis is not always corresponded because of the lack of the adequate diagnosis methods. Leira et al. (Leira et al. 2008) show that stroke management practices in rural areas are suboptimal, which creates an unacceptable health disparity between urban patients with stroke and their rural counterparts. The main problem in rural areas is that a rapid diagnosis cannot be achieved, hence an early treatment cannot be administered. Possible solutions are related to education of caregivers and the increase of state resources. Other solutions include the use of $\mathrm{AI}$ in helping to make earlier diagnosis procedure.

The handling of medical decisions concerning stroke type diagnosis by using AI techniques is not a new approach. During the last two decades the problem has been primarily faced using different machine learning algorithms. Alexopoulos et al. (Alexopoulos, Dounias, and Vemmos 1999) used the C4.5 algorithm by building a decision tree. These authors reported that inductive machine learning is a promising approach for computer-aided diagnosis of stroke. Linder et al. (Linder et al. 2006) used logistic regression and artificial neural networks for classifying acute ischemic stroke. They concluded that logistic regression was the best algorithm for the classification of acute ischemic stroke. However, the comparison was only performed between two algorithms. There are other approaches based on machine learning that try to predict stroke subtypes (Arslan, Colak, and Sarihan 2016). Zhang et al. (Zhang et al. 2013), on the other hand, tried to find correlations between physiological parameters of stroke patient during 48 hours after stroke, and their stroke outcomes after three months, predicting degrees of disability symptoms in Modified Rankin Scale (0 $=$ no significant disability, $1=$ slight disability, $\ldots, 6=$ death).

The aforementioned previous works elaborated AI models based on many features. These techniques require many patients and many variables to train the models (a relatively short and wide input data table). In this direction, Djatna et al. (Djatna, Hardhienata, and Masruriyah 2018) tried to find out the minimal set of features able to diagnose the type of stroke using fuzzy logic. The set of features was reduced from 40 to 25 using the International Stroke Trial Database, which includes data on 19435 patients.

However, our purpose is not to use a big set of static features (as a relatively short and wide input data table of 19435 records of 25 features), but to use a set of non invasive permanent monitoring data (a very long and narrow input data table, with more than $1.5 \times 10^{6}$ records of 7 features) that could be measured even in ambulatory environments. The reason is that it is easy and not expensive to obtain. Non invasive monitoring data also provide enough information in a short period of time, and can be easily acquired in rural areas. In this paper, we propose an alternative solution based on the diagnosis of the illness with a computer aided approach, using machine learning techniques. Our technique consists in determining the stroke type using a short time window of non-invasive data. These data can be easily collected, since any ambulatory unit is equipped with the necessary infrastructure to measure these data. Our results show that we are able to recognize the stroke type with a $92 \%$ of accuracy. Besides, our methodology is able to predict possible medical complications, currently tested with the prediction of an eventual death, with a $96 \%$ of accuracy, respectively. Being aware of a high risk of death will allow intensive surveillance in order to avoid possible imminent complications and apply preventive treatments. Another aspect of managing high risk mortality patients is being able to inform family with a more accurate prognosis. 
Our work, however, not only performs a detailed comparison among diagnosis algorithms to detect the stroke types, but also a comparison among different predictive algorithms to determine possible subsequent complications. Both sides -diagnosis and prediction techniques- have been uniformly designed, since are always based on non-invasive data.

\section{METHODS}

\subsection{Dataset}

To perform this study a Philips PIIC iX monitoring center was installed in the Stroke Care Unit of the Hospital Universitario de La Princesa. This device was collecting data from March 2017 to July 2017 from all the patients admitted to the Stroke Care Unit during this period. This included data from 119 stroke patients (105 ischemic and 14 hemorrhagic). The definition of all the non-invasive variables obtained through the monitoring station is summarized in Table 1 . These variables were periodically measured, with a period equal to 30 seconds. At the end of these 3 months, a set of more than $1.8 \times 10^{6}$ data points were collected.

Table 1: Definition of the variables employed in the current study.

\begin{tabular}{ccccc}
\hline Variable & Abbreviation & Type & Role & Description \\
\hline Type of Stroke & TS & Categorical & Ischemic/Hemorrhagic & Target \\
Risk Prediction & RP & Categorical & Exitus/Non Exitus & Target \\
Rhythm Estimation & RE & Numerical & Input & A Rhythm Indicator \\
EV & EV & Numerical & Input & Ventricular extra systole \\
FC & FC & Numerical & Input & Cardiac Frequency \\
Breathing Frequency & BF & Numerical & Input & Respiratory Rate \\
Perf & - & Numerical & Input & Pulmonar Perfusion \\
SpO2 & - & Numerical & Input & Oxygen Saturation \\
ST-II & - & Numerical & Input & Systolic Time Interval Index \\
\hline
\end{tabular}

\subsection{Preprocessing of the dataset}

Before starting the classification process, it was necessary to process the dataset to prepare it for classification. Since the dataset has been elaborated from measurements acquired by sensors in a clinical environment, data incoherences happen due to a bad connection or a punctual malfunctioning of the sensors. Therefore, the first step consisted on detecting and removing outliers. Therefore, those values that were greater than four times the standard deviation of each variable were replaced by its mean value.

Apart from outliers, null values, i.e. variables that were not recorded, needed a special treatment. This was done in two ways, depending on the type of the variable. In variables related to cardiac frequency, each null value was replaced by the value measured in the previous time interval. For the rest of the variables, the null values of numerical variables were replaced with the mean values of the valid measures obtained within the time window.

The second step consisted of normalization. Since the features are in different ranges between them, a normalization process is needed before classification. For each variable, a Z-Standardization was performed. This standardization consists of subtracting the mean value $(\mu)$ of each variable and divide the result by the standard deviation of the variable $(\sigma)$. Therefore, each variable $\mathrm{X}$ is converted into a new variable $\mathrm{Z}$ 
following the next formula:

$$
\mathrm{Z}=\frac{X-\mu}{\sigma}
$$

\subsection{Machine learning algorithms}

In the following, we briefly describe the set of machine learning algorithms used in this study. All of them have been used through the Scikit-Learn Python library (Pedregosa, Varoquaux, Gramfort, Michel, Thirion, Grisel, Blondel, Prettenhofer, Weiss, Dubourg, Vanderplas, Passos, Cournapeau, Brucher, Perrot, and Duchesnay 2011).

\subsubsection{Decision Tree}

Decision Trees are a non-parametric supervised learning method used for classification and regression. The goal is to create a model that predicts the value of a target variable by learning simple decision rules inferred from the data features. Decision Trees are simple to understand and to interpret, the cost of using the tree (i.e., predicting data) is logarithmic, and performs well even if its assumptions are violated by the true model from which the data were generated.

\subsubsection{K-Nearest Neighbors $(\mathrm{K}-\mathrm{NN})$}

The K-nearest neighbors algorithm (K-NN) is a non-parametric method. The input consists of the K closest training examples in the feature space. The output depends on whether K-NN is used for classification or regression. In K-NN classification, which is the case of this study, the output is a class membership. An instance is classified by a majority vote of its neighbors, with the instance being assigned to the class most common among its $\mathrm{K}$ nearest neighbors. $\mathrm{K}$ is a positive integer, typically small.

\subsubsection{Logistic Regression (LR)}

Logistic Regression is a linear model used for classification in which the probabilities describing the possible outcomes are modeled using a logistic function. This model aims to solve an optimization problem with different objective functions. In this study, a L1 regularized logistic regression was solved through a Newtoncg solver.

\subsubsection{Naive Bayes}

Naive Bayes is a family of supervised learning algorithms based on applying Bayes' theorem with the "naive" assumption of independence between every pair of features. Given a class variable $y$ and a dependent feature vector $x_{1} \ldots x_{n}$, by applying the Bayes' theorem the following classification rule can be reached:

$$
\begin{aligned}
P\left(y \mid x_{1}, \ldots, x_{n}\right) & \propto P(y) \prod_{i=1}^{n} P\left(x_{i} \mid y\right) \\
& \Downarrow \\
\hat{y} & =\underset{y}{\operatorname{argmax}} P(y) \prod_{i=1}^{n} P\left(x_{i} \mid y\right),
\end{aligned}
$$


and Maximum A Posteriori (MAP) estimation can be used to estimate $P(y)$ and $P\left(x_{i} \mid y\right)$. The former is the relative frequency of class $y$ in the training set.

\subsubsection{Neural Network}

Artificial neural networks are a computational structure that try to emulate the actual interconnection between biological neural networks and how they interact. There are several types of artificial neural networks. In this study, because of the nature of the problem, we have selected the multi-layer perceptron network. It is a supervised learning algorithm that learns a function $f(\cdot): R^{m} \rightarrow R^{n}$ by training on a dataset, where $m$ is the number of dimensions for input and $n$ is the number of dimensions for output. Given a set of features $X=x_{1}, x_{2}, \ldots, x_{m}$ and a target $y$, it can learn a non-linear function approximator for either classification or regression.

\subsubsection{Random Forest}

Random forests or random decision forests are an ensemble learning method. They are typically used for classification or regression. A random forests operate by constructing a multitude of decision trees at training time. When used in classification, the output is the class that is the mode of the classes of the individual trees. The purpose of random decision forests is to correct the overfitting habit of decision trees to their training set.

\subsubsection{Support Vector Machines (SVM)}

SVM is a supervised learning approach for classification and regression tasks. It is utilized in order for linear/nonlinear classification problems with high-dimensional datasets. To solve a nonlinear classification problem, SVM maps the input sets to a high-dimensional space by applying various kernel functions. A detailed explanation of SVM approach can be achieved in (Bishop 2006). The advantage of this kernel is that it transforms data that are not linearly separable in their original vector space into another space that could be highly linearly separable, which makes the classification much more accurate and less resourceconsuming.

\subsection{Experimental setup}

To evaluate the performance of each model, a 10-fold cross validation resampling method was used. This process guarantees the independence of the validation set from the training one and the validity of the trained classifier against any unseen sample. The objective was to obtain unbiased outputs and to avoid the overfitting problem. A seed number was randomly fixed and used for each data partition process. This procedure ensures the use of the same training and testing data in the modeling process. The Python library Scikit-Learn was used to run all the algorithms.

The model performance evaluation metrics used to compare the set of algorithms proposed were: accuracy, sensitivity, specificity, FMeasure and both the areas under the Receiver Operating Characteristic (ROC) curve and the Precision Recall Curve (PRC). These metrics are defined as follows:

$$
\text { Accuracy }=\frac{\mathrm{TP}+\mathrm{TN}}{\mathrm{TP}+\mathrm{TN}+\mathrm{FP}+\mathrm{FN}},
$$




$$
\begin{gathered}
\text { Sensitivity }=\text { Recall }=\frac{\mathrm{TP}}{\mathrm{FN}+\mathrm{TP}}, \\
\text { Specificity }=\frac{\mathrm{TN}}{\mathrm{FP}+\mathrm{TN}}, \\
\mathrm{F}-\text { Measure }=\frac{2 \cdot \text { Precision } \cdot \text { Recall }}{\text { Precision }+ \text { Recall }},
\end{gathered}
$$

where Precision $=\mathrm{TP} /(\mathrm{TP}+\mathrm{FP})$, TP is the number of true positives, $\mathrm{TN}$ is the number of true negatives, FP is the number of false positives, and FN is the number of false negatives (Hernández-Orallo, Flach, and Ferri 2012).

\section{RESULTS}

Initially, outlying data were removed and, since the measured variables exhibit different magnitudes, the dataset was normalized through Z-normalization as stated above. After that we trained, validated and tested our two models (stroke subtype diagnosis and exitus prediction) with the data belonging to the first 8 hours of admission of all the 119 patients.

In the following, we present all the results obtained for each model type: stroke type diagnosis and death prediction.

\subsection{Stroke subtype diagnosis}

From the 119 patients monitored during three months, 14 were diagnosed at hospital as hemorrhagic and 105 as ischemic, which perfectly fits the normal proportion of ischemic strokes.

Table 2 shows the detailed results of the performance metrics of each algorithm tested. The last row shows the average of all the six metrics. These average values were 0.8921 for Decision Trees, 0.8708 for KNearest Neighbors, 0.6518 for Logistic Regression, 0.6515 for Naive Bayes, 0.5606 for Neural Networks, 0.9295 for Random Forest and 0.6079 for Support Vector Machines. As Table 2 illustrates, the Random Forest algorithm was the best one on average. Indeed, this algorithm was the best in 5 out of the 6 metrics. The neural network was the only better than the Random Forest in the Recall metric. However, NN classified all the strokes as ischemic, being the NN Recall metric equal to 1, the NN Specificity metric equal to 0, and offering a low performance in the other 4 metrics.

Figure 1 depicts a graphical representation of all the performance metrics of each model together with the averaged value.

\subsection{Death prediction}

In this case, the methodology included, although completely equivalent, the computation of a predictive model. The model will calculate, after eight hours of monitoring, if the patient will be able to overcome the stroke or not.

Table 3 shows the detailed results of the performance metrics of each algorithm tested. As in the previous Section, the last row shows the average of all the six metrics. These average values were 0.9390 for Decision 
Table 2: Stroke subtype diagnosis: performance metrics.

\begin{tabular}{cccccccc}
\hline Perf. metric & Decision Tree & K-NN & Logistic Regression & N. Bayes & NN & Random Forest & SVM \\
\hline Accuracy & 0.8897 & 0.8607 & 0.6374 & 0.6089 & 0.4980 & $\mathbf{0 . 9 1 9 9}$ & 0.6000 \\
Recall & 0.9262 & 0.9034 & 0.6279 & 0.9923 & $\mathbf{1 . 0 0 0 0}$ & 0.9385 & 0.5307 \\
Specificity & 0.8535 & 0.8184 & 0.6469 & 0.2284 & 0.0000 & $\mathbf{0 . 9 0 1 4}$ & 0.6687 \\
F-Measure & 0.8932 & 0.8660 & 0.6330 & 0.7165 & 0.6649 & $\mathbf{0 . 9 2 1 1}$ & 0.5692 \\
ROC Area & 0.8899 & 0.8609 & 0.6374 & 0.6104 & 0.5000 & $\mathbf{0 . 9 2 0 0}$ & 0.5997 \\
PRC Area & 0.9000 & 0.9154 & 0.7285 & 0.7524 & 0.7010 & $\mathbf{0 . 9 7 5 8}$ & 0.6791 \\
Avg. & 0.8921 & 0.8708 & 0.6518 & 0.6515 & 0.5606 & $\mathbf{0 . 9 2 9 5}$ & 0.6079 \\
\hline
\end{tabular}

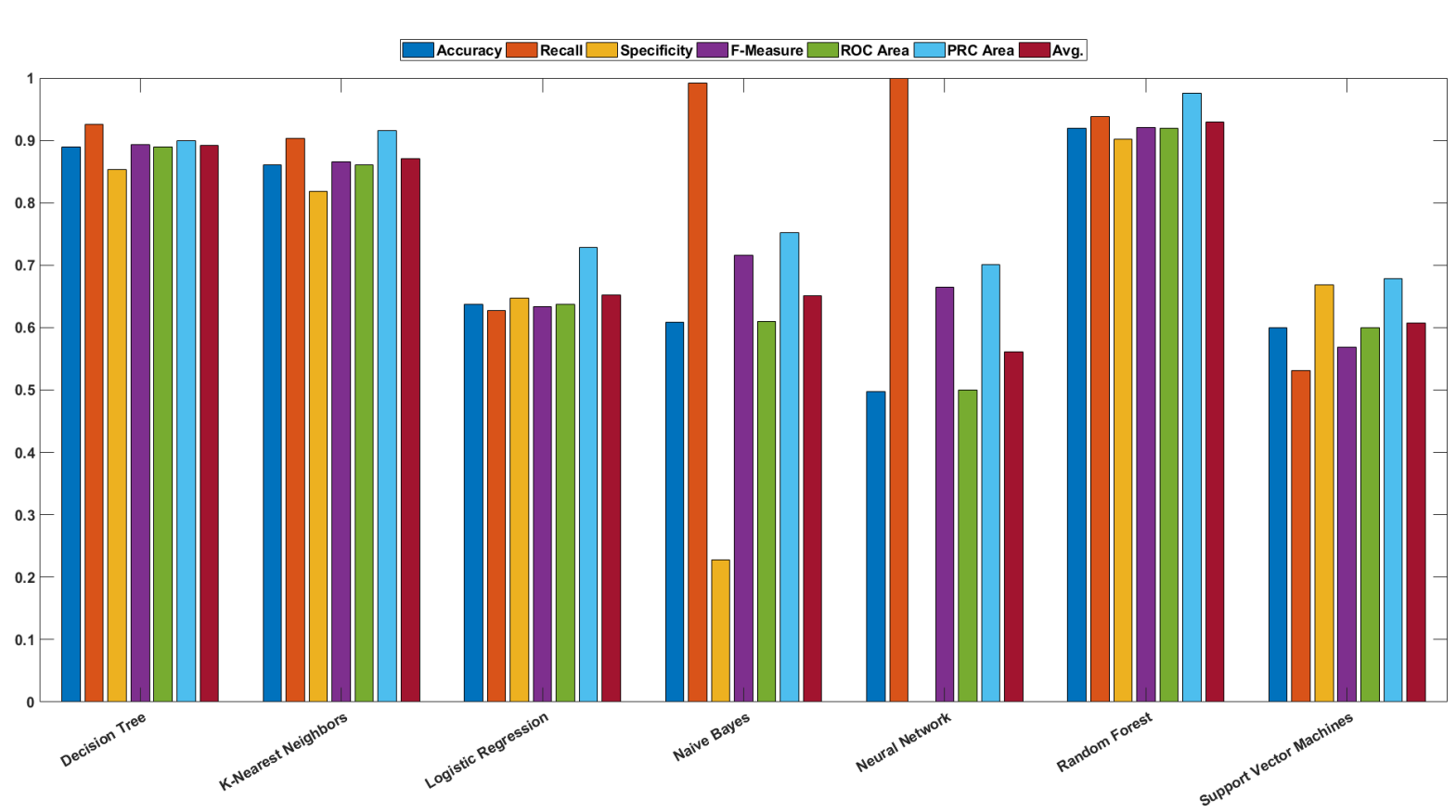

Figure 1: Stroke subtype diagnosis: all the performance metrics of each model together.

Trees, 0.9371 for K-Nearest Neighbors, 0.7513 for Logistic Regression, 0.7175 for Naive Bayes, 0.4527 for Neural Networks, 0.9673 for Random Forest and 0.9092 for Support Vector Machines. As Table 2 illustrates, the Random Forest algorithm was the best one on average, reaching the best value in 4 of 6 metrics. As in the previous model, the neural network did not reach a good balance of true and false predictions. Thus, it obtained a perfect value for the Specificity metric, but the worst in Recall. SVM obtained the best value for Recall, but not too far of the value obtained by the Random Forest model.

Figure 2 depicts a graphical representation of all the performance metrics of each model together with the averaged value.

\section{CONCLUSIONS AND FUTURE WORK}

Medical data mining is one application area where both diagnostic and predictive models can be accurately obtained. To this end, performance metrics are very important to evaluate the quality of the models (Arslan, Colak, and Sarihan 2016).

In this paper we have developed this idea around the diagnosis of the stroke type (hemorrhagic vs ischaemic) and the prediction of further complications of the disease. 
Table 3: Exitus prediction: performance metrics.

\begin{tabular}{cccccccc}
\hline Perf. metric & Decision Tree & K-NN & Logistic Regression & N. Bayes & NN & Random Forest & SVM \\
\hline Accuracy & 0.9362 & 0.9306 & 0.7404 & 0.7013 & 0.4996 & $\mathbf{0 . 9 6 1 9}$ & 0.9056 \\
Recall & 0.9332 & 0.9547 & 0.7655 & 0.8006 & 0.0000 & 0.9566 & $\mathbf{0 . 9 7 2 9}$ \\
Specificity & 0.9392 & 0.9065 & 0.7152 & 0.6020 & $\mathbf{1 . 0 0 0 0}$ & 0.9673 & 0.8382 \\
F-Measure & 0.9361 & 0.9323 & 0.7469 & 0.7284 & 0.0000 & $\mathbf{0 . 9 6 1 7}$ & 0.9116 \\
ROC Area & 0.9362 & 0.9306 & 0.7403 & 0.7013 & 0.5000 & $\mathbf{0 . 9 6 1 9}$ & 0.9055 \\
PRC Area & 0.9531 & 0.9682 & 0.7992 & 0.7714 & 0.7165 & $\mathbf{0 . 9 9 4 5}$ & 0.9212 \\
Avg. & 0.9390 & 0.9371 & 0.7513 & 0.7175 & 0.4527 & $\mathbf{0 . 9 6 7 3}$ & 0.9092 \\
\hline
\end{tabular}

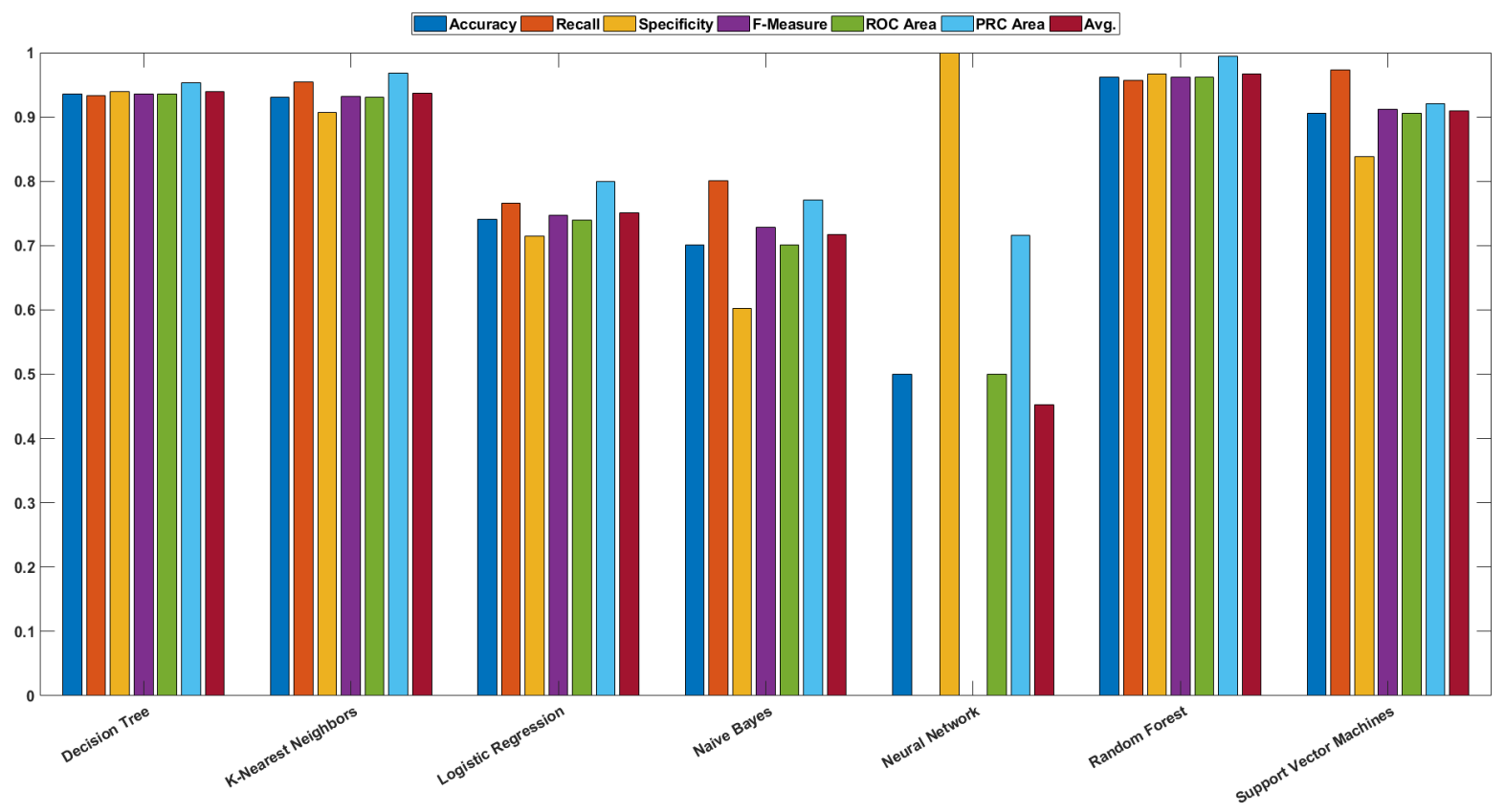

Figure 2: Exitus prediction: all the performance metrics of each model together.

Being able to identify ischaemic stroke is critical in order to provide early and accurate treatment. But this is not always possible due to the lack of diagnostic resources, such brain image scan like CT or MRI, specially in rural areas. We have tested 7 well known algorithms to define diagnostic models, which based on non-invasive data, are able to correctly identify hemorrhagic vs ischaemic stroke with a $92 \%$ of accuracy.

We have also tested these 7 algorithms to define predictive models. In particular, we have trained models to predict critical events after the stroke like the eventual death, with an accuracy of $96 \%$. To reach these results, we have performed a 10-fold cross validation test.

Our current study has revealed that computer-aided approaches such as machine learning over medical data are an effective instrument in the diagnosis of the stroke type, but also in the prediction of risks. Obtaining early diagnostic information is crucial in special medical settings: pre-hospital medical evaluation, lack of imaging scan or late access due to long geographic distances, as it happens in rural areas.

Having an instrument that allows the prediction of medical complications, like mortality, gives the medical team the opportunity to react early and rapidly start a treatment. In some occasions, this will allow doctors to use preventive treatments in order to avoid the oncoming adversity. In addition to the medical treatment, 
having a prediction model of outcome would allow doctors to give more accurate information to the patient or the patient's family about stroke risks and prognosis.

As part of our future work, we are planning to integrate both the data acquisition process and the predictive model into a small and wearable hardware device, simplifying the life of both patients and physicians. We are also integrating the prediction of other relevant risks, like rebleeding.

\section{REFERENCES}

Alexopoulos, E., G. D. Dounias, and K. Vemmos. 1999. "Medical diagnosis of stroke using inductive machine learning". In Machine learning and applications, pp. 20-23.

Arslan, A. K., C. Colak, and M. E. Sarihan. 2016. "Different medical data mining approaches based prediction of ischemic stroke". Computer Methods and Programs in Biomedicine vol. 130, pp. 87-92.

Bishop, C. M. 2006. Pattern Recognition and Machine Learning (Information Science and Statistics). Secaucus, NJ, USA, Springer-Verlag New York, Inc.

Djatna, T., M. K. D. Hardhienata, and A. F. N. Masruriyah. 2018, Oct. "An intuitionistic fuzzy diagnosis analytics for stroke disease". Journal of Big Data vol. 5 (1), pp. 35.

Haidong Wang, e. a. 2016. "Global, regional, and national life expectancy, all-cause mortality, and causespecific mortality for 249 causes of death, 1980-2015: a systematic analysis for the Global Burden of Disease Study 2015”. The Lancet vol. 388 (10053), pp. 1459-1544.

Hernández-Orallo, J., P. Flach, and C. Ferri. 2012, October. "A Unified View of Performance Metrics: Translating Threshold Choice into Expected Classification Loss". J. Mach. Learn. Res. vol. 13 (1), pp. 2813-2869.

Hill, M. D. 2005. "Diagnostic Biomarkers for Stroke: A Stroke Neurologist's Perspective". Clinical Chemistry vol. 51 (11), pp. 2001-2002.

Jiang, F., Y. Jiang, H. Zhi, Y. Dong, H. Li, S. Ma, Y. Wang, Q. Dong, H. Shen, and Y. Wang. 2017. "Artificial intelligence in healthcare: past, present and future". Stroke and Vascular Neurology vol. 2 (4), pp. 230243.

Kanchana, R., and R. Menaka. 2017. "A novel approach for characterisation of ischaemic stroke lesion using histogram bin-based segmentation and gray level co-occurrence matrix features". The Imaging Science Journal vol. 65 (2), pp. 124-136.

Leira, E. C., D. C. Hess, J. C. Torner, and H. P. A. Jr.. 2008. "Rural-urban differences in acute stroke management practices: A modifiable disparity". Archives of Neurology vol. 65 (7), pp. 887-891.

Linder, R., I. König, C. Weimar, H. Diener, S. Pöppl, and A. Ziegler. 2006. "Two models for outcome prediction". Methods Inf. Med. vol. 45, pp. 536-540.

Pedregosa, F., G. Varoquaux, A. Gramfort, V. Michel, B. Thirion, O. Grisel, M. Blondel, P. Prettenhofer, R. Weiss, V. Dubourg, J. Vanderplas, A. Passos, D. Cournapeau, M. Brucher, M. Perrot, and E. Duchesnay. 2011. "Scikit-learn: Machine Learning in Python". Journal of Machine Learning Research vol. 12, pp. 2825-2830.

World Health Organization 2018. "The top 10 causes of death".

Zhang, Q., Y. Xie, P. Ye, and C. Pang. 2013. "Acute ischemic stroke prediction from physiological time series patterns". Australasian Medical Journal vol. 6 (5), pp. 280-286. 


\section{AUTHOR BIOGRAPHIES}

LUIS GARCÍA-TERRIZA is a Ph.D. candidate at the Complutense University of Madrid (UCM). His work focuses on the development of robust modeling and simulation methodologies for the behavior of neurological diseases, developing models for prompt prediction and classification of critical events. His email address is luigar05@ucm.es.

JOSÉ L. RISCO-MARTÍN received his Ph.D. from Complutense University of Madrid, and currently is Associate Professor in the Department of Computer Architecture and Automation at Complutense University of Madrid. His research interests include computer aided design, optimization and discrete event simulation. His email address is jlrisco@ucm.es.

GEMMA REIG ROSELLÓ is a Ph.D. candidate at the Stoke Care Unit of the Hospital Universitario de La Princesa, where she is Area Specialist in Neurology since 2009. She can be reached at gemmareig@hotmail.com.

JUAN MIGUEL CAMARASALTAS is the Healthcare IT technician at the IT Department of Hospital Universitario de La Princesa. He can be reached at juanmiguel.camarasaltas@salud.madrid.org.

JOSÉ L. AYALA got his PhD in Electronic Engineering from Technical University of Madrid and is currently an Associate Professor in the Department of Computer Architecture and Automation at Complutense University of Madrid. His research interests focus on IoT and edge solutions for personalized medicine approaches, including health monitoring, wireless sensor networks and disease modeling. His email address is jayala@ucm.es. 